\title{
A Sustained Complete Response in an Elderly Patient with Metastatic Colorectal Adenocarcinoma-A Clinical Case
}

\author{
Maria Leitão, Isabel Vilas-Boas, Manuela Machado* \\ Medical Oncology Department, Portuguese Institute of Oncology of Porto, Porto, Portugal \\ Email: *m.machado.fn@gmail.com
}

How to cite this paper: Leitão, M., Vilas-Boas, I. and Machado, M. (2020) A Sustained Complete Response in an Elderly Patient with Metastatic Colorectal Adenocarcinoma-A Clinical Case. Journal of Cancer Therapy, 11, 351-356.

https://doi.org/10.4236/jct.2020.116029

Received: April 27, 2020

Accepted: May 26, 2020

Published: May 29, 2020

Copyright (c) 2020 by author(s) and Scientific Research Publishing Inc. This work is licensed under the Creative Commons Attribution International License (CC BY 4.0).

http://creativecommons.org/licenses/by/4.0/

\begin{abstract}
Background: The increasing life expectancy of people worldwide will signify that oncologists everywhere will be faced with many difficult decisions regarding treatment options in the elderly population with cancer. These patients carry specific challenges that should be discussed. Aim: To report the clinical case of an elderly patient with a metastatic colorectal cancer with a sustained complete response and reflect on the particularities on this special population. Clinical case: In 2009, an 80-year-old man presents with colorectal cancer, undergoes surgery and intraoperatively a peritoneal implant is resected. He is deemed fit for chemotherapy and is treated with 8 cycles of capecitabine. After a 10-year follow-up, he remains with no evidence of disease. Conclusion: Chronological age is insufficient to establish a treatment plan; oncologists must evaluate the patient multidimensionally and develop personalized options for the therapy and follow-up of older individuals.
\end{abstract}

\section{Keywords}

Elderly, Chemotherapy, Cancer, Colorectal

\section{Introduction}

The developed world has witnessed a growing percentage of older people in the last decades and even developing countries are beginning to see a shift in the demographic pyramid. Modern medicine, hygienic improvements and economic stability have contributed to an escalating life expectancy.

Simultaneously, the incidence and prevalence of cancer is on the rise worldwide. Cancer is the main cause of death among those who are 60 to 79 years old [1]. It follows that oncologists are increasingly faced with older patients carrying 
oncological disease in need of treatment, especially considering that the incidence of many cancers increases with age (e.g. colorectal cancer, which is more commonly diagnosed in patients 65 to 74 years old) [2]. Despite this fact, older adults are still underrepresented in most clinical trials, including trials on target therapies and immunotherapy.

This section of the population is a heterogeneous group with unique issues. Many people above 65 years old maintain an active lifestyle and have the potential to enjoy full lives for decades to come. Therefore, chronological age should not be the only factor in therapeutic decisions. However, there may be biological differences in response to treatment and side effects in this population. Concerns about amplified toxicity of chemotherapy and diminished gains when it comes to overall survival and disease free survival justify the fact that these patients are offered chemotherapy treatment less often than their younger counterparts (when they are referred to a medical oncologist at all) [3]. The existence of multiple comorbidities, more common in an ageing population, affects colorectal cancer survival in retrospective analyses [4].

There are several models and tools of geriatric assessment available to guide physicians in this conundrum. The likely life expectancy regardless of oncological disease should be taken into account, as well as the patients' functional status, comorbidities, organ function, physical mobility, cognitive function, nutritional status, availability of social support and economic issues. Considerations should also be made on the oncological disease (type of tumor, stage, burden of disease) and the type and related toxicities of the possible treatment options. Attention should also be paid to the patients' concurrent medications (polypharmacy). The goals and expectations of the patient should, as always, be integrated into the therapeutic plan [5].

Therapy may have curative intent and palliative intent. The latter offers the perspective of improved survival, control of symptoms, higher quality of life and preservation of functional status. These are valuable goals even for those in their last decades.

The authors report a clinical case that highlights the issues of the management of oncological disease in this setting. The patient gave consent to the writing and publication of this information.

\section{Clinical Case}

The authors present the case of a patient of a man, born in June 1929. In July of 2009, he complained of abdominal pain to his family doctor. The appropriate tests were solicited and a fecal occult blood test turned out positive. The colonoscopy revealed a mass located in the sigmoid region of the colon, which was biopsied. In October of 2009, at 80 years old, a diagnosis of colorectal adenocarcinoma is obtained. A CT scan of the thorax, abdomen and pelvis was requested, showing no evidence of distant disease (Figure 1).

The patient was submitted to an anterior rectal resection with simultaneous 


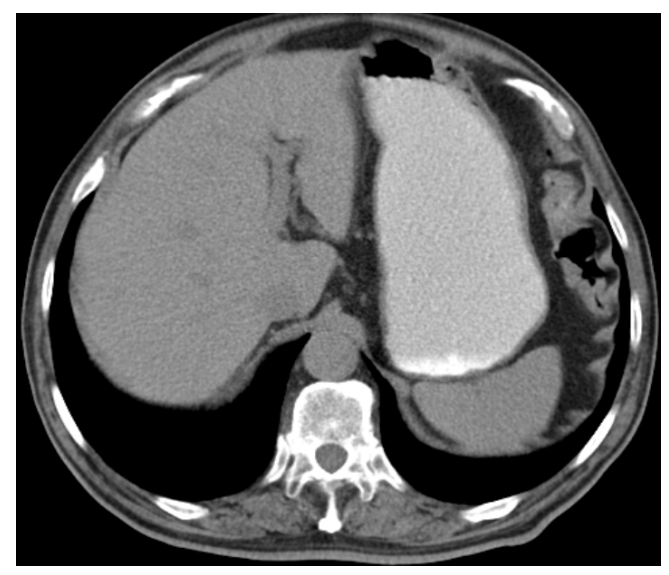

Figure 1. CT scan of the abdominal, metastasis absent.

excision of a peritoneal implant found intra-operatively. The histology of the surgical specimen revealed an adenocarcinoma of the rectosigmoid transition, grade 2, pT3N2 (with 7 metastasized lymph nodes with extracapsular invasion of 27 excised) M1, with no involved margins-R0 surgery; it was also positive for lymphovascular invasion and perineural invasion. On a later analysis, no mutation of proteins MLH1, MSH2, MSH6 and PMS2 was found; therefore, the tumor was classified as microsatellite-stable (MSS).

A Medical Oncology consultation was required. At this time, the patient was completely asymptomatic and his performance status (PS) was compatible with ECOG PS 1. He was independent for his activities of daily living and instrumental activities of daily living. He suffered from arterial hypertension as his only medical comorbidity. He had also been submitted some years prior to a left total knee replacement, had undergone prostatic surgery at 40 years old as well as surgical treatment of a duodenal ulcer at 32 years old. On analytical tests, he showed normal hematological function, Grade 1 total bilirubin elevation, creatinine clearance of $45 \mathrm{ml} / \mathrm{min}$ (according to Cockcroft-Gault formula) [6] and a CEA of $3.38 \mathrm{ng} / \mathrm{ml}$ (normal range $<4 \mathrm{ng} / \mathrm{ml}$ ). He was proposed for chemotherapy with capecitabine $\left(1250 \mathrm{mg} / \mathrm{m}^{2}\right.$ bid day 1 to 14 ; cycles of 21 days).

In 2010 , he completed 8 cycles of chemotherapy with capecitabine ( 6 months total). He demonstrated good tolerance to the treatment, without any relevant side effects.

Later in the year 2010, he underwent a total colonoscopy, a CT scan and laboratory testing with CEA and CA19.9, which were all within normal parameters.

During follow-up he needed a pacemaker placement in 2015, suffered two ischemic strokes in 2016 and in 2019 he developed stage 4 chronic kidney disease.

On his last CT scan (thoraco-abdomino-pelvic) in 2012 there was no evidence of recurrence. His last colonoscopy in 2014 found no lesions. On his last imaging exams, an abdominal ultrasound and a thoracic radiography, in 2015, none revealed suspected images.

The patient expressed his wish of not performing further follow-up radiographic exams, unless symptoms arose, maintaining clinical follow-up thence- 
forth.

Currently, in April 2020, he is 90 years old. The patient shows signs of neurologic impairment, without any other symptoms and maintaining an ECOG PS 1. His last CEA test was $2.24 \mathrm{ng} / \mathrm{ml}$.

In conclusion, the patient has no evidence of disease, after a 10 years follow-up of a stage IV colorectal adenocarcinoma on an elderly patient.

\section{Discussion}

This case report raises the question if chemotherapy should be proposed after R0 surgery of a stage IV colorectal cancer in an asymptomatic elderly patient and, if so, which regime should be used.

The physicians involved considered that the biological age of the patient was younger than his chronological age, which, allied to his low burden of disease and relative lack of comorbidities, made him a suitable candidate for chemotherapy. Additionally, even though it seemed to be totally removed, peritoneal disease carries a poor prognosis and the possibility of cure after resection is not as well established as in liver metastases [7]. Therefore, and in consonance with the recommendations for complementary chemotherapy after resection of liver and lung metastases, treatment with capecitabine was deemed beneficial. Fluoropirimidine regimens have proved to be valuable in the adjuvant treatment of older patients [8]. Oxaliplatin has shown reduced benefit in overall survival and disease free survival when added to fluoropyrimidines in adjuvant stage III colorectal cancer in patients above 70 years old, including in subset analyses of the NSABP C-07 and MOSAIC trials [9] [10] [11]. However, this patient had a peritoneal implant removed during surgery and FOLFOX could be considered in the palliative/perioperative setting, even in patients older than 70 years old, although sometimes controversial [3] [12]. The first clinical trial on older adults in the palliative setting failed to achieve benefit in overall survival with the addition of oxaliplatin to fluoropyrimidine regimens [13] and a pooled safety analysis found higher haematological toxicity with FOLFOX in older patients [8].

The decision was made due to the better tolerance of capecitabine monotherapy and easier administration (oral versus intravenous) in a patient with no measurable disease. In fact, retrospective analyses demonstrate that this is a frequent treatment choice for older adults with metastatic colon cancer [14].

An important caveat would be that at the time of diagnosis, presence of RAS/BRAF mutations was not regularly researched in clinical practice and thus its result is unknown in this case, with its potential predictive and prognostic implications, but biologic agents are not usually recommended in the complementary chemotherapy after R0 resection of metastatic disease [5].

Another topic of discussion would be how follow-up should be carried out in an ageing population. In an elderly patient with resected M1 disease should we conform to the usual guidelines or individualize the plan? If a personalized plan is conceived, which exams to order and how frequently? 
In the present report, the follow-up was planned in accordance with the wishes of the patient. At first, regular visits to the clinic were required, scheduled at progressively longer intervals. Consultations became annual after 5 years of follow-up. The first years of follow-up, CT scans were solicited every 3 - 6 months in agreement to the recommendations. Later, more innocuous exams were preferred (ultrasound and chest X-ray), until finally the assistant physician and the patient agreed on forgoing any more imaging exams.

In the advent of the COVID-19 pandemic, it was decided to conduct the clinical consultation by telephone, to protect the patients' fragile health. In April of 2020, the patient was well and his granddaughter mentioned that he maintains agricultural activities.

This case underlines the fundamental importance of considering the special characteristics of this population and of adapting the existing evidence to the reality of each situation. Without reflection on these variables, blindly applying guidelines will result in increased morbility and toxicity; simply limiting access to therapies with regard only to chronological age will increase mortality and lower quality of life.

\section{Conclusion}

The elderly represent a special population, with different challenges and particularities. Patients above 70 years old should be offered a geriatric evaluation with multidimensional assessment, which, coupled with the patients' individual goals and expectations, should help guide treatment and follow-up decisions. Doctor-patient communication is key in a balanced and holistic strategy, tailored for the specific individual.

\section{Conflicts of Interest}

The authors declare no conflicts of interest regarding the publication of this paper.

\section{References}

[1] Siegel, R.L., et al. (2018) Cancer Statistics, 2018. CA: A Cancer Journal for Clinicians, 68, 7-30. https://doi.org/10.3322/caac.21442

[2] National Cancer Institute. Surveillance, Epidemiology, and End Results Program: SEER Fact Sheets-Colon and Rectum Cancer. http://seer.cancer.gov/statfacts/html/colorect.html

[3] McCleary, N.J., Dotan, E. and Browner, I. (2014) Refining the Chemotherapy Approach for Older Patients with Colon Cancer. Journal of Clinical Oncology, 32, 2570-2580. https://doi.org/10.1200/JCO.2014.55.1960

[4] Gross, C.P., Guo, Z., McAvay, G.J., Allore, H.G., Young, M. and Tinetti, M.E. (2006) Multimorbidity and Survival in Older Persons with Colorectal Cancer. Journal of the American Geriatrics Society, 54, 1898-1904. https://doi.org/10.1111/j.1532-5415.2006.00973.x

[5] NCCN Guidelines in Older Adult Oncology, Version 2.2020. 
[6] Cockcroft, D.W. and Gault, M.H. (1976) Prediction of Creatinine Clearance from Serum Creatinine. Nephron, 16, 31-41. https://doi.org/10.1159/000180580

[7] Franko, J., et al. (2016) Prognosis of Patients with Peritoneal Metastatic Colorectal Cancer Given Systemic Therapy: An Analysis of Individual Patient Data from Prospective Randomised Trials from the Analysis and Research in Cancers of the Digestive System (ARCAD) Database. The Lancet Oncology, 17, 1709-1719. https://doi.org/10.1016/S1470-2045(16)30500-9

[8] Goldberg, R.M., et al. (2006) Pooled Analysis of Safety and Efficacy of Oxaliplatin plus Fluorouracil/Leucovorin Administered Bimonthly in Elderly Patients with Colorectal Cancer. Journal of Clinical Oncology, 24, 4085-4091.

https://doi.org/10.1200/JCO.2006.06.9039

[9] McCleary, N.J., et al. (2013) Impact of Age on the Efficacy of Newer Adjuvant Therapies in Patients with Stage II/III Colon Cancer: Findings from the Accent Database. Journal of Clinical Oncology, 31, 2600-2606.

[10] Yothers, G., et al. (2011) Oxaliplatin as Adjuvant Therapy for Colon Cancer: Updated Results of NSABP C-07 Trial, Including Survival and Subset Analyses. Journal of Clinical Oncology, 29, 3768-3774. https://doi.org/10.1200/JCO.2011.36.4539

[11] Tournigand, C., et al. (2012) Adjuvant Therapy with Fluorouracil and Oxaliplatin in Stage II and Elderly Patients (between Ages 70 and 75 Years) with Colon Cancer: Subgroup Analyses of the Multicenter International Study of Oxaliplatin, Fluorouracil, and Leucovorin in the Adjuvant Treatment of Colon Cancer Trial. Journal of Clinical Oncology, 30, 3353-3360. https://doi.org/10.1200/JCO.2012.42.5645

[12] Nordlinger, B., et al. (2008) Perioperative Chemotherapy with FOLFOX4 and Surgery versus Surgery Alone for Resectable Liver Metastases from Colorectal Cancer (EORTC Intergroup Trial 40983): A Randomised Controlled Trial. The Lancet, 371, 1007-1016. https://doi.org/10.1016/S0140-6736(08)60455-9

[13] Seymour, M.T., Thompson, L.C., Wasan, H.S., et al. (2011) Chemotherapy Options in Elderly and Frail Patients with Metastatic Colorectal Cancer (MRC FOCUS2): An Open-Label, Randomised Factorial Trial. The Lancet, 377, 1749-1759. https://doi.org/10.1016/S0140-6736(11)60399-1

[14] Ho, C., Ng, K., O’Reilly, S., et al. (2005) Outcomes in Elderly Patients with Advanced Colorectal Cancer Treated with Capecitabine: A Population-Based Analysis. Clinical Colorectal Cancer, 5, 279-282. https://doi.org/10.3816/CCC.2005.n.040 